\title{
Pensiero, immagini mentali e creatività in diversi stati di vigilanza: il contributo della scuola di Marcello Cesa-Bianchi
}

\section{Thinking, mental imagery, and creativity in different states of vigilance: The contribution of Marcello Cesa-Bianchi school}

\author{
Alessandro Antonietti* e Carlo Cipolli ${ }^{\circ}$ \\ * Dipartimento di Psicologia, Università Cattolica del Sacro Cuore, Milano \\ L.go A. Gemelli, 1, 20123 Milan, Italy \\ e-mail: alessandro.antoniett@unicatt.it; tel: 027234.2284 \\ - Dipartimento di Medicina Specialistica, Diagnostica e Sperimentale, \\ Università di Bologna \\ Via Giuseppe Massarenti 9, 40138 Bologna, \\ e-mail: carlo.cipolli@unibo.it; telefono: 0512091816
}

Ricevuto: 25.06.2020 - Accettato: 30.09.2020

Pubblicato online: 07.05.2021

\section{Riassunto}

Marcello Cesa-Bianchi ha svolto un ruolo importante nel favorire gli studi sperimentali degli psicologi italiani sulla funzione delle immagini visive all'interno del processo di pensiero creativo durante i diversi stati di vigilanza (veglia vigile, veglia rilassata, rêverie, addormentamento e sonno REM). Egli era partito dalla constatazione che la ricerca di soluzioni innovative per nuovi problemi artistici, scientifici o di vita quotidiana richiede una ricombinazione flessibile e creativa di alcune conoscenze pregresse. Questo articolo riporta le prove sperimentali a sostegno sia dell'assunto che le immagini mentali generate e trasformate intenzionalmente possono facilitare la soluzione dei problemi, sia delle successive ipotesi su come le strategie innovative e intuitive di manipolazione delle immagini mentali possano operare durante la veglia e altri stati di vigilanza. Dopo aver delineato sinteticamente i risultati degli studi in condizioni di veglia a sostegno di una stretta relazione tra la capacità di manipolare mentalmente le immagini in modo olistico e il pensiero creativo, vengono riportati i risultati di vari studi recenti i quali mostrano che l'intuizione (insight) che preannuncia la soluzione può avvenire durante il sonno così come nell'addormentamento, nella veglia rilassata e

A. Antonietti \& C. Cipolli. / Ricerche di Psicologia, 2021, Vol. 44(1),

ISSN 0391-6081, ISSNe 1972-5620, Doi: 10.3280/rip1-2021oa11597

Copyright $\odot$ FrancoAngeli

This work is released under Creative Commons Attribution - Non-Commercial No Derivatives License. For terms and conditions of usage please see: http://creativecommons.org 
nella rêverie (mind wandering). Tutti questi stati di vigilanza favoriscono sia la diffusione dell'attivazione di nuclei di conoscenza episodica e semantica nelle reti associative (coinvolte anche nella produzione di sogni durante il sonno), sia la ristrutturazione della rappresentazione del problema attraverso il trasferimento di relazioni tra diversi tipi di informazioni. Soprattutto il sonno REM può funzionare come un periodo di incubazione in grado di aumentare i tassi di soluzione di compiti come la scoperta di regole matematiche nascoste e la risoluzione di anagrammi. Quasi tutti i risultati sperimentali confermano l'idea che un periodo di sonno - così come di veglia rilassata, rêverie o addormentamento - facilita la soluzione di problemi indipendentemente dalle loro caratteristiche artistiche, scientifiche o di vita quotidiana.

Parole chiave: creatività, immagini mentali, soluzione di problemi, incubazione, stati di vigilanza, sogno, sonno

\section{Summary}

Marcello Cesa-Bianchi played an important role in fostering the experimental studies of Italian psychologists on the function of visual images within the process of creative thinking during distinct states of vigilance (wakefulness, resting state, sleep onset, and REM sleep). He moved from the observation that finding innovative solutions for novel artistic, scientific, or everyday-life problems requires a flexible and creative recombination of some items of prior knowledge. This paper outlines the items of experimental evidence supporting his presupposition that intentionally generated and transformed mental images can facilitate the solution of problems and the subsequent hypotheses as to how innovative and intuitive strategies of manipulating mental images can work during wakefulness and other states of vigilance. After sketching the findings of studies in waking supporting a close relationship between the ability to mentally manipulate images in a holistic way and creative thinking, the findings of several recent studies showing that an insight heralding the solution may occur during sleep, resting, mind wandering, and sleep onset are reported. All these states of vigilance foster both spreading activation of items of episodic and semantic knowledge along associated networks (involved also in dream generation during sleep) and restructuring of problem representation through a transfer of relationships between different types of information. Overall REM sleep may work as an incubation period capable to enhance solution rates in discovery of hidden mathematical rules and solving anagrams. Almost all the items of evidence converge to support the notion that a period of sleep - as well of resting, mind wandering, or sleep onset - is beneficial for solving problems regardless of their artistic, scientific, or everydaylife characteristics.

Keywords: creativity, mental imagery, problem solving, incubation, vigilance states, dreaming, sleep 


\section{Premessa}

La psicologia del pensiero nella scuola di Marcello Cesa-Bianchi è stata sviluppata in varie direzioni, in relazione sia ai temi (p. es. per l'intelligenza si rimanda al contributo di Amoretti, 2021) che alle diverse fasi del ciclo di vita (per l'età evolutiva si rimanda al contributo di Albanese, Farina e Fiorilli, 2021, e per l'età anziana al contributo di Cristini, G. CesaBianchi e De Beni, 2021) e con diverse modalità (ricerche di allievi, collaborazioni con istituti e centri di ricerca nazionali e internazionali, promozione di linee di ricerca emergenti attraverso riviste e congressi).

In questo articolo si intende soffermarsi su una particolare e originale linea di ricerca, quella delle valenze funzionali del pensiero a base visiva in diversi stati di vigilanza (ovvero veglia, sonno e stati intermedi, resting e stati ipnagogici). La linea di ricerca è stata coltivata a lungo presso l'Istituto di Psicologia della Facoltà Medica dell'Università degli Studi di Milano sia direttamente che in collegamento con le ricerche sui processi mentali del sonno sviluppate nelle Università di Bologna e di Roma-Sapienza, i cui risultati erano già apprezzati a livello internazionale. Il collegamento era stato favorito dall'interesse di Cesa-Bianchi per lo studio degli stati crepuscolari e ipnagogici, anche a fini psicoterapici.

Sul versante "interno" questa linea si innestava sulla prospettiva teorica elaborata da Enrico Barolo, che presso l'Istituto di Psicologia della Facoltà Medica dell'Università di Milano agli inizi degli anni ' 80 del secolo scorso aveva cercato di individuare forme alternative, rispetto a quelle previste dalla teoria piagetiana (nell'ambito evolutivo) e dal cognitivismo classico (nell'ambito della psicologia cognitiva), attraverso le quali i processi di ragionamento si attuano nella mente umana (Barolo, 1981). In questo ambito l'immagine mentale si segnalava come una forma di rappresentazione che può non soltanto svolgere un ruolo di supporto al pensiero astratto o a base verbale, ma anche permettere di compiere operazioni precluse a quest'ultimo (in particolare nell'area della generazione di idee inusuali e innovative) o comunque costituire una strategia complementare al pensiero logico o sostitutiva (in certe circostanze addirittura più efficace di quest'ultimo).

In questa area di ricerca si era inserito Roberto Masini, il cui principale interesse era rivolto allo studio della percezione, ma che si era anche occupato, sulla scia della notorietà che in Italia avevano acquisito le indagini di Paivio sul valore di immagine delle parole (Cornoldi, 1976), dell'immagine mentale (Masini, 1979). Questa prospettiva aveva incuriosito due studenti della Scuola di Specializzazione in Psicologia dell'Università degli Studi di Milano, che furono con generosa disponibilità accolti nel gruppo. Uno (Paolo Stramba-Badiale) avrebbe poi privilegiato lo studio delle fun- 
zioni dell'immagine mentale - nella forma soprattutto delle rêverie - nelle dinamiche emotive, con applicazioni nel campo della psicologia clinica (Barolo e Stramba-Badiale, 1989) (verso il quale Barolo stava orientando principalmente i propri interessi), diventando noto psicoanalista e fondando una propria scuola di psicoterapia. L'altro (coautore di questo contributo) avrebbe proseguito la ricerca in questa area (e.g., Antonietti, Cerana e Scafini, 1994) approfondendo il ruolo delle differenze individuali (e.g., Antonietti, Bologna e Lupi, 1997) e della metacognizione (e.g., Antonietti, 1999), anche in combinazione con dati neurobiologici (e.g., Incorpora, Oliveri, Genevini, Santagostino, Tettamanti, Antonietti e Risoli, 2010; Oldrati, Colombo e Antonietti, 2018). Le ricerche condotte tra la metà degli anni '80 e l'inizio degli anni '90 da questo "quartetto" - in cui le (talvolta ingenue) congetture dei due esuberanti giovani venivano riportate al rigore della sperimentazione dai due maestri, non mancando di aggiornare periodicamente il Direttore dell'Istituto, il quale non lesinava utili suggerimenti - sono l'oggetto della ricostruzione presentata nella prima parte di questo testo. Va ricordato che la tematica delle funzioni delle immagini mentali ha trovato ospitalità su questa rivista anche successivamente (Antonietti e Di Nuovo, 2014), grazie all'interesse per questo argomento da parte di Santo Di Nuovo (1999), che ebbe intense collaborazioni con Cesa-Bianchi.

Sul versante esterno, già negli anni '70 Cesa-Bianchi aveva manifestato interesse per le ricerche sperimentali sulle attività mentali del sonno in varie occasioni, inserendo workshops e sezioni tematiche in congressi da lui organizzati o presieduti e dedicando un numero monotematico di $R i$ cerche di Psicologia ai "Processi mentali durante il sonno" (1980). Già nel titolo, che andava oltre la caratterizzazione al momento dominante (psicofisiologia del sonno e del sogno), veniva individuata un'area molto estesa di ricerca, quella dei meccanismi cognitivi ed emozionali coinvolti nelle numerose attività mentali documentate dalle ricerche neurofisiologiche degli anni ' $60-$ ' 70 in diversi stati di vigilanza. Ciascuno dei 10 capitoli del volume, aperto da una "Introduzione" memorabile per coerenza teorica e competenza epistemologica (Bosinelli, 1980), sintetizzava le principali evidenze acquisite in uno specifico ambito di ricerca e suggeriva sviluppi resi possibili anche da paradigmi nuovi o rivisitati dai ricercatori delle Università di Bologna e di Roma-Sapienza.

Il gruppo di Bologna, nel quale si stava inserendo un co-autore di questo contributo, aveva sviluppato a partire dagli ultimi anni '60 nel nuovo Istituto di Psicologia della Facoltà Medica varie ricerche, focalizzate soprattutto sulle modificazioni psicofisiologiche nelle fasi di addormentamento (stadio I e II del sonno NREM) rispetto sia alla veglia che al sonno profondo (stadi III-IV di sonno NREM) e al sonno REM. Grazie a conso- 
lidati collegamenti internazionali (da parte di Marino Bosinelli e Sergio Molinari con centri di ricerca negli Stati Uniti, da parte di Piero Salzarulo con centri di ricerca a Parigi e a Lione) le ricerche realizzate erano al passo con gli orientamenti teorici e le metodiche di data analysis più avanzati del momento. L'ampia gamma di metodiche disponibili permise di studiare sia le caratteristiche di specifici contenuti (come quelli verbali) delle attività mentali del sonno REM e NREM (Salzarulo e Cipolli, 1974), sia i processi di memoria dei contenuti delle attività mentali del sonno REM e NREM (encoding durante il sonno e recall dopo il risveglio: Salzarulo e Cipolli, 1979; Cipolli, 1980), sia i processi di elaborazione di specifiche informazioni trasmesse prima del sonno (pre-sleep stimuli) e incorporate tra i contenuti delle attività mentali durante sonno REM e NREM (Cipolli et al., 1983). I risultati quest'ultima linea di ricerca entravano pleno jure tra le evidenze potenzialmente rilevanti per la comprensione delle relazioni fra immagini mentali e creatività nel sonno.

\section{Il ruolo delle immagini mentali nella soluzione di problemi}

Alla fine degli anni ' 70 i risultati delle ricerche sui processi di elaborazione durante il sonno di informazioni acquisite nella veglia precedente apparivano rilevanti anche per la comprensione delle relazioni fra immagini mentali e creatività per due ragioni. In primo luogo, le immagini mentali, bandite dal comportamentismo, erano state prima "riammesse" tra gli oggetti di studio della psicologia scientifica negli anni '60 e poi pienamente "riabilitate" dall'orientamento cognitivista degli anni '70 (Mehler e Bever, 1972). Dopo i tentativi iniziali di determinarne sperimentalmente la natura - ovvero, se siano rappresentazioni mentali simil-percettive (soprattutto visive: Shepard, 1975) o astratte (codificate in un linguaggio proposizionale: Anderson, 1978) - si stava optando per accertarne la funzione svolta in vari processi cognitivi, come la memoria, l'apprendimento e il pensiero. In secondo luogo, un ruolo importante delle immagini mentali nel pensiero creativo era da sempre accreditato da una copiosa aneddotica di scienziati e artisti, che riportavano di avere ricevuto l'intuizione (insight) decisiva per scoperte e opere d'arte in forma visivo-figurale o uditiva (per opere musicali) in stati di ridotta attenzione (decentramento cognitivo) o addirittura in fasi di sonnolenza o durante il sonno.

Le ricerche sperimentali dei 40 anni successivi hanno permesso di accertare le funzioni svolte nel pensiero creativo da fattori sia psicologici (come la riduzione delle interferenze a seguito di un'interruzione del compito, la riorganizzazione delle informazioni disponibili ecc.) sia neurofisiologici (caratterizzanti gli stati di vigilanza: veglia attiva, veglia rilassata o resting state, mind wandering, addormentamento, sonno profondo, son- 
no REM), consentendo di analizzare più analiticamente il ruolo delle immagini mentali. Per certi versi lo studio delle relazioni tra immagini mentali e creatività (o soluzione creativa di problemi) in diversi stati di vigilanza può essere considerato emblematico della fertilità euristica dell'approccio a un fenomeno psicologicamente ben definito attraverso linee di ricerca apparentemente molto distanti.

L'indagine dei rapporti tra rappresentazioni mentali di tipo visivo e funzioni euristiche del pensiero costituiva all'inizio degli anni ' 80 un campo di ricerca scarsamente - e mai sistematicamente - esplorato. Anche se una lunga tradizione aneddotica e una frequente pratica didattica suggerivano che i modelli visivi rivestono un'utile funzione nell'ambito della scoperta e della risoluzione di problemi di ordine intellettivo, erano relativamente poche le indagini volte a precisare i fattori che influiscono sull'impiego produttivo delle rappresentazioni visivo-spaziali.

Perché, innanzi tutto, indagare le funzioni dell'immagine mentale nella soluzione di problemi? La cultura occidentale ha in genere sottovalutato le potenzialità del pensiero visivo, tendenzialmente considerato o come un pensiero che precede lo sviluppo di modi di ragionamento che delle immagini possono poi fare a meno (una sorta di "trampolino di lancio" per il pensiero astratto) o come una sorta di "stampella" cui il pensiero astratto ricorre quando si trova in difficoltà (per esempio, quando occorre spiegare un concetto a una persona per la quale è difficile seguire delle dimostrazioni logiche). In altre culture però non è così, come segnalavano etnologi e psicologi transculturali (Inghilleri, 2009). Per esempio, in certe popolazioni nomadi i pastori si rendono conto della mancanza nel gregge di qualche capo non contando gli animali ad uno ad uno, ma attraverso una semplice "occhiata" lanciata al gregge: una funzione che la nostra scuola ci ha abituato a svolgere attraverso una procedura matematica è qui assolta attraverso una più rapida procedura intuitivo-visiva (Gladwell, 2007).

È ampiamente documentato che di strategie visivo-spaziali ci si avvale anche per risolvere problemi complessi. Per esempio, per gli abitanti delle isole polinesiane l'orientamento nella navigazione è stabilito per mezzo di modelli mentali di tipo spaziale, anziché - come avviene nella scienza nautica occidentale - attraverso un complesso sistema di calcoli. Vi sono poi popoli che dedicano un'attenzione del tutto particolare a quella forma particolare di pensiero per immagini che è costituito dai contenuti dei "sogni" (ovvero, come hanno dimostrato le ricerche di laboratorio, $i$ contenuti delle attività mentali ricordate al mattino, molto spesso elaborate in corrispondenza di prolungati periodi di sonno REM: Snyder, 1970). In alcune tribù della Malesia centrale, come i Senoi, il racconto e l'elaborazione dei sogni costituisce una parte rilevante dell'educazione della gioventù. Ogni mattina, prima i bambini e poi gli adulti, raccontano i sogni della notte. In 
seguito gli uomini si riuniscono in un consiglio nel quale vengono ripresi e discussi i sogni più impressionanti. Lo scopo di questa pratica (molto prossima alla tecnica psicoterapica del dream sharing) è quello di aiutare chi ha compiuto un sogno in cui si sono manifestati elementi negativi (paura, odio, incidenti, lutti) a sfruttare tali esperienze per volgerle, nella realtà, verso mete positive. Infatti, chi ha raccontato il sogno che successivamente è stato oggetto di discussione viene invitato a risognarlo in modo diverso durante il giorno, in uno stato di rilassamento. Da questo secondo "viaggio onirico" il sognatore deve tornare con qualcosa di creativo che possa essere comunicato agli altri: un'azione da compiere, un'ispirazione per un prodotto artistico (una poesia, un canto, una danza, una scultura, un racconto), la soluzione di un problema (Shepard, 1975).

Dal complesso di suggestioni cui si è fatto cenno emerge l'indicazione che il pensiero a base visiva può risultare efficace per la soluzione dei problemi. Perché ciò avviene? Si possono individuare differenti ragioni (Antonietti, 1991). Innanzi tutto, le rappresentazioni visive, secondo la prospettiva allora sostenuta da Kosslyn (1980), sono rappresentazioni analogiche della realtà; esse mantengono cioè alcune delle caratteristiche spaziali del mondo e degli oggetti che esse riproducono. Ciò comporta che quando operiamo mentalmente su di un'immagine, questa ci permette di mantenere un rapporto di isomorfismo con le situazioni reali. Pertanto, se il rapporto con la realtà è particolarmente importante, l'immagine ci permette di operare in modo più produttivo rispetto ai simboli logico-verbali, che implicano invece un maggior livello di astrazione e un maggior numero di mediazioni concettuali.

Ancora, le rappresentazioni figurali permettono di estrapolare dalle situazioni problemiche le caratteristiche più importanti, producendo quindi una visione schematica in grado di evidenziare la struttura del problema o della situazione. Vi sono casi in cui la rappresentazione mentale in forma visiva, proprio grazie al carattere di strutturalità, offre notevoli vantaggi. Tale rappresentazione permette infatti di estrapolare rapidamente dalle informazioni gli elementi pertinenti e di evidenziare i tratti essenziali.

Le rappresentazioni mentali di tipo visivo possono poi essere trasformate in maniera olistica, ossia agendo contemporaneamente su tutte le loro parti e non parte dopo parte. In altre parole, il codice visivo permette l'attuazione di una sorta di raggruppamento od ostensione simultanea di dimensioni che diversamente non sarebbero rappresentabili se non attraverso una procedura sequenziale. In questo modo le rappresentazioni di tipo visivo-spaziale favoriscono l'elaborazione delle informazioni perché, permettendo di compiere più operazioni simultaneamente, producono un risparmio di lavoro mentale. Inoltre, attraverso le immagini mentali possiamo eseguire valutazioni in modo rapido, in quanto esse ci aiutano a co- 
gliere immediatamente i rapporti proprio perché esse permettono di rappresentare ed elaborare le informazioni "in parallelo", ossia in modo che siano tenuti contemporaneamente in considerazione vari aspetti. Infine, la possibilità di tenere presenti più aspetti simultaneamente è particolarmente utile quando si tratta di operare nell'ambiguità e nell'incertezza, ossia in situazioni in cui non è opportuno scegliere subito una direzione ed escludere le altre, ma è meglio considerare tutti i possibili percorsi dato che non si hanno indizi sicuri circa la preferibilità dell'uno o dell'altro. Su questa base risulta comprensibile perché la visualizzazione favorisce modi di procedere di tipo intuitivo, permettendo di giungere rapidamente e facilmente alle conclusioni senza dover compiere passaggi intermedi o senza dover utilizzare strategie ripetitive.

Le rappresentazioni figurali hanno anche la capacità di simulare la situazione problemica in un modo facilmente manipolabile. Le immagini sono infatti rappresentazioni estremamente flessibili. Esse permettono, inoltre, di immaginare alcune trasformazioni degli elementi del problema che risulterebbero impossibili da attuare nella realtà. Il problema, infatti, per poter essere risolto, talvolta deve essere impostato in modo diverso da quello in cui esso si presenta immediatamente. Si devono prospettare altre sue possibili articolazioni e l'immagine fungerebbe proprio da rappresentazione anticipatoria di queste articolazioni alternative. Attraverso la visualizzazione si può trapassare da una situazione all'altra o compiere delle repentine associazioni, anche tra elementi (apparentemente) molto distanti tra loro. Per la combinazione delle immagini non esiste infatti una sintassi normativa: le combinazioni possono essere le più diverse. Anzi, le giustapposizioni insolite sono in genere quelle che dischiudono sorprese o novità. La capacità di combinare degli elementi - in questo caso immagini - in modi inconsueti o di scorgere aspetti interessanti nei risultati di tali combinazioni è infatti considerata una delle componenti della creatività.

In una serie di indagini di tipo correlazionale (raccolte in Antonietti, Barolo e Masini, 1988) si è mostrato come l'abilità di risolvere in modo innovativo problemi di tipo matematico, geometrico, verbale e pratico è connessa a specifiche attitudini all'elaborazione immaginativa. In particolare, il pensiero produttivo è risultato connesso a operazioni di trasformazione mentale di immagini di tipo olistico e strutturale; al contrario, operazioni immaginative di tipo sequenziale sono connesse alle capacità di pensiero logico-astratto ma non al problem solving creativo. Inoltre, la vividezza e la precisione delle immagini mentali sono risultate, negli adulti come nei bambini, di ostacolo alla ristrutturazione dei problemi. Più in specifico, le soluzioni di tipo creativo dei problemi emergono in coloro che hanno buona capacità di compiere la sintesi immaginativa, ossia di combinare mentalmente figure parziali per costruire un'immagine com- 
plessiva (Antonietti, Barolo e Masini, 1985). L'abilità di trasformare le figure nell'immaginazione attraverso operazioni di tipo sequenziale (come i ribaltamenti, che implicano una serie di passaggi) non risulta invece particolarmente correlata alla capacità di risolvere problemi che implicano la ristrutturazione della rappresentazione iniziale, capacità che è invece collegata alla campo-indipendenza (Antonietti, Barolo e Masini, 1986). Se, tuttavia, queste trasformazioni, come la rotazione, vengono compiute in maniera olistica e non parte per parte o passaggio dopo passaggio, l'abilità immaginativa è in relazione alle capacità di problem solving produttivo, $\mathrm{e}$ questa relazione è ravvisabile anche in soggetti non vedenti (Barolo, Masini e Antonietti, 1990).

In queste indagini, tra l'altro, venne ideato un compito di composizione di immagini mentali che andava oltre le prove allora esistenti in cui le parti da collegare tra loro non cambiavano significato una volta inserite nel prodotto finale della sintesi (Thompson e Klatzky, 1978). Nella sintesi mentale messa a punto le parti delle figure di partenza, una volta combinate in un'unica figura, assumevano un diverso ruolo. Per esempio i segmenti che nelle immagini di partenza erano le ali di una freccia nell'immagine-sintesi diventavano i lati di un rombo. Si mostrava così, benché questo risultato non venne messo in luce appieno, che anche nell'immaginazione vigono principi di organizzazione figurale del tutto analoghi a quelli della percezione, come si cercava di provare tentando di riprodurre con immagini mentali note illusioni ottiche.

\section{Prime indicazioni sul ruolo delle immagini mentali nel sonno per la soluzione di problemi}

I presupposti teorici e le prime evidenze delle ricerche sperimentali sulle relazioni fra immagini mentali e creatività in diverse condizioni di vigilanza sono descritti in vari articoli del già citato numero monotematico di Ricerche di Psicologia (1980). Infatti, vari articoli (due dei quali scritti da David Foulkes e Milton Kramer, tra i più eminenti studiosi di psicofisiologia del sonno) documentavano l'esistenza in un'area molto più estesa di ricerca rispetto a quella individuabile attraverso la semplicistica dicotomia tra attività mentale "oniro-simile" e "pensiero-simile" che era stata proposta alla fine degli anni '50 dopo la scoperta dell'organizzazione ciclica del sonno. Tale scoperta era stata accompagnata dal riscontro della presenza di attività mentali caratterizzate nel sonno REM (acronimo di $\mathrm{ra}$ pid eye movements sleep) da contenuti prevalentemente visuo-percettivi, spesso bizzarri ed emozionalmente intensi, e negli altri stadi di sonno (corrispondenti all'addormentamento e al sonno lento, ovvero gli stadi 1-2 e 3-4 del sonno NREM) da contenuti poco vividi e riferibili ad eventi e 
preoccupazioni o problemi della vita quotidiana. Tale area riguardava $\mathrm{i}$ meccanismi cognitivi ed emozionali coinvolti nelle numerose attività mentali documentate dalle ricerche neurofisiologiche degli anni ' $60-$ ' 70 in vari stati di vigilanza.

Questa semplicistica dicotomia, tuttavia, era stata rapidamente superata a seguito del riscontro di frequenti attività mentali di breve durata, ma con caratteristiche visuo-percettive vivide anche in addormentamento (stadio 1 e 2 NREM), definibili come "sogni di addormentamento" (Foulkes, 1962). Negli anni successivi venne definitivamente documentato che i contenuti delle attività mentali del sonno a) sono in parte iterati in stadi di sonno sia della stessa notte (Rechtschaffen, Vogel e Shaikun, 1963) che di notti successive (Kramer e Roth, 1979); b) tendono a diventare più oniro-simili nella seconda parte della notte, indipendentemente dallo stadio di sonno (Snyder, 1970); c) spesso incorporano (cioè, riattivano ed elaborano: replay) informazioni acquisite prima del sonno e da utilizzare dopo il risveglio per compiti di memoria (Foulkes e Rechtschaffen, 1964), a volte anticipandone la esecuzione (o "soluzione"). Quest'ultimo tipo di indicazioni rese evidente l'analogia tra la facilitazione esercitata dal replay durante il sonno di informazioni relative ad un compito da completare dopo il risveglio e l'effetto Zeigarnik (Zeigarnik, 1927) di soluzione differita di compiti interrotti. La rivisitazione dell'effetto Zeigarnik come risultato anche di processi cognitivi durante il sonno (concepito come stato non eminentemente passivo, ma caratterizzato da attività mentali con caratteristiche ricorrenti e prevedibili) rappresentava verso la fine degli anni '70 una sfida stimolante per studiosi dotati di competenze sia psicofisiologiche che cognitiviste.

L'analogia ipotizzabile tra i processi cognitivi coinvolti nelle attività mentali del sonno e quelli sottostanti l'insight nella veglia era suffragata anche dal riscontro che nelle attività mentali elaborate in "veglia rilassata" (resting: Foulkes e Fleisher, 1975) o in rêverie ipnagogica (ottenuta con semi-deprivazione sensoriale e somministrazione di white-noise: Bertini, Lewis e Witkin, 1964) compaiono spesso alcuni contenuti oniro-simili (soprattutto visuo-percettivi).

Nel volume Processi mentali durante il sonno il saggio di Bertini (1980), oltre a riassumere i risultati delle ricerche sviluppate in prima persona fin dagli anni ' 60 sulle caratteristiche delle attività mentali del sonno di soggetti con diversi stili percettivi (campo-dipendenti vs campo-indipendenti, secondo la tipologia di Witkin e Lewis), segnalava la possibilità di cogliere nella rêverie ipnagogica elementi di continuità sia con la vita "diurna" che con i processi attivi nel problem solving. A tale proposito Bertini rimarcava l'importanza delle immagini mentali (visual imagery) elaborate in una condizione sperimentale simile all'addormentamento, in 
quanto le loro caratteristiche richiamavano spesso l'evoluzione della rappresentazione iniziale di un problema fino all'insight nella condizione di veglia.

La fertilità euristica delle implicazioni di questa rivisitazione dell'effetto Zeigarnik (1927) come risultato di processi cognitivi attivi anche in stati di vigilanza diversi dalla veglia si è manifestata pienamente solo a distanza di quasi due decenni. Tuttavia numerosi dati sia sperimentali che empirici hanno contribuito a renderla sempre più plausibile, mostrando che la soluzione di specifici compiti cognitivi (anagrammi, associazioni su informazioni verbali, etc.) è facilitata dopo periodi di sonno rispetto a periodi di veglia (Walker et al., 2002). Inoltre, accanto all'aneddotica di soluzioni di problemi apparse in sogno nel corso della notte (o in addormentamento) a scienziati ed artisti, è stata documentata una frequenza non trascurabile di soluzioni di problemi della vita quotidiana "anticipate" in sogni di persone comuni (cfr. le rassegne di Barrett, 1993 e 2001).

La compatibilità di dati di laboratorio e (auto)osservazioni empiriche ha finalmente portato nei primi anni '2000 a rigorose verifiche sperimentali dell'influenza positiva del sonno sulla soluzione di problemi complessi, ovvero di tipo logico-matematico.

\section{L'elaborazione attiva di informazioni nel sonno}

Le prime rigorose dimostrazioni sperimentali dell'attivazione durante il sonno di alcuni processi coinvolti nel problem solving sono state ottenute misurando il funzionamento di specifici processi cognitivi correlati con le prestazioni "creative" nei brevi periodi di sleep inertia successivi a risveglio in sonno REM e NREM, allorché persistono le caratteristiche neurofisiologiche e cognitive dello stadio di sonno precedente (cfr. la rassegna di Tassi e Muzet, 2000).

Una maggiore flessibilità cognitiva dopo risveglio in sonno REM rispetto a NREM, oltre che rispetto alla veglia, si è riscontrata anzitutto nell'accesso a informazioni lessicali: l'attivazione di relazioni associative "deboli" (per es, tra "auto" e "mela") risulta essere sensibilmente più rapida dopo sonno REM (soprattutto nella seconda parte della notte) rispetto sia al sonno NREM che alla veglia (Stickgold et al., 1999). Invece, il sonno NREM in genere facilita l'estrazione di tratti semantici comuni da diverse informazioni e, quindi, l'integrazione di informazioni recenti con altre remote e/o astratte (Lewis, Knoblich e Poe, 2018). Questi dati spiegano sia la maggiore bizzarria (ovvero "imprevedibilità" su base semantica) dei contenuti delle AMS nella seconda parte della notte (con prevalente sonno REM), che la comparsa di insight prolungati verso il mattino (come spesso riferito da artisti, per esempio Tartini per la composizione del "Il trillo 
del diavolo"). Inoltre, la presenza di numerosi contenuti interrelati, cioè simili, in distinte AMS della stessa notte (Cipolli et al., 2006) e di notti successive (Nielsen, 2004) evidenzia l'accesso ripetuto (replay) alle stesse informazioni episodiche e semantiche, favorendo l'esplorazione delle loro relazioni associative e, quindi, la "scoperta" di analogie insospettate rispetto a quelle già focalizzate nella veglia. Invece, il sonno NREM in genere facilita l'estrazione di tratti semantici comuni da diverse informazioni e, quindi, l'integrazione di informazioni recenti con altre remote e/o astratte (Lewis et al., 2018).

Invece, per compiti di tipo matematico (come il Number Reduction Task) che richiedono di "scoprire" un regola nascosta (hidden), il sonno REM risulta più favorevole rispetto sia alla veglia che al sonno NREM (Wagner et al., 2004). Infatti, il sonno REM facilita la scomposizione di concetti pre-esistenti e la ricombinazione (rebinding) di alcuni loro elementi con altri in una nuova "rappresentazione" mentale, che supera le restrizioni auto-imposte (mental set) nell'utilizzo delle informazioni disponibili. Inoltre, anche per compiti semantici astratti (come il Remote Associates Test) il sonno REM esercita un'influenza più positiva rispetto al sonno NREM (Cai et al., 2009): coerentemente con la maggior durata del sonno REM nella seconda parte della notte, l'insight si manifesta più spesso verso il mattino.

\section{Incubazione delle informazioni rilevanti dei problemi e creatività du- rante il sonno}

I risultati di molte ricerche successive hanno evidenziato che non tutti i tipi di compito beneficiano allo stesso modo del sonno, anche se in esso sono comunque agevolati in quanto non esposti alle interferenze di stimoli esterni o di attività concomitanti. Il sonno è piuttosto un periodo di "incubazione", che facilita l'insight in modo diverso a seconda sia del tipo di sonno (REM/ NREM) che del compito da svolgere (Lewis et al., 2018). Già le consegne trasmesse ai soggetti (così come le abituali "intenzioni" codificate nella memoria prospettica, ovvero le attività da svolgere dopo il risveglio: per una rassegna, cfr. Barner et al., 2017; Leong et al., 2019) fanno variare sensibilmente la probabilità di incorporare un'informazionestimolo (come una frase da ricordare o un compito da completare dopo il risveglio) tra i contenuti delle attività mentali soprattutto del sonno REM (Cipolli et al., 2001, 2004; Schoch et al., 2019). Nel sonno (almeno) REM è presente qualche forma di controllo attivo, pur se non volontario, delle informazioni da selezionare in memoria per poi trasformarle in contenuti di attività mentali, facendo emergere l'esistenza di relazioni inusuali o in- 
sospettate fra di loro. Tali relazioni favoriscono l'insight, che spesso evidenzia non un'analogia fra due elementi, ma un insieme di relazioni fra vari elementi trasferibile da un contesto ad un altro.

I dati sperimentali acquisiti negli ultimi anni appaiono complessivamente coerenti anche con le indicazioni implicite in molte auto-descrizioni degli insight alla base di scoperte scientifiche e di opere artistiche (Barrett, 2001). Le parziali discrasie riscontrate sembrano dipendere per lo più da tre limitazioni metodologiche delle ricerche sperimentali finora realizzate: a) l'utilizzo di compiti standardizzati anziché contestualizzati (e differenziati) in rapporto all' expertise dei soggetti (come avviene necessariamente nelle attività artistiche e scientifiche); b) la misurazione degli effetti del sonno nella sola notte successiva alla presentazione del compito (anziché in più notti successive: nella vita quotidiana l'insight a volte compare dopo settimane o mesi); c) la messa a fuoco degli effetti sull'insight indotti dal sonno (REM/ NREM) anziché dell'addormentamento (stadio 1- 2 di sonno NREM) e di altri stati di vigilanza potenzialmente importanti per la creatività come il resting (Cai et al., 2018; Li et al., 2016) e il mind wandering (Gable, Hopper e Schooler, 2019; Leszczynski et al., 2017).

Al momento non vi sono dati sperimentali sufficienti per stabilire se anche nelle immagini ipnagogiche (i cosiddetti sogni di addormentamento) compaia un insight di tipo divergente, poi rielaborato in stadi e cicli successivi della stessa notte. L'interesse teorico di questa ipotesi consiste in due ordini di fatti. Anzitutto, vi è una certa continuità nei contenuti delle attività mentali elaborate in addormentamento e in quelle precedenti al risveglio del mattino (Cipolli et al., 2003). In secondo luogo, i resoconti autobiografici forniti da artisti e scienziati differiscono spesso nella descrizione del momento dell'insight. Le opere artistiche hanno richiesto di solito un insight prolungato (di tipo convergente) relativo alla struttura complessiva dell'opera (come un poema o una composizione musicale), che è intervenuto nel sonno (presumibilmente REM) della seconda parte della notte. Gli insight che hanno condotto a scoperte scientifiche, invece, sono comparsi spesso durante l'addormentamento sotto forma di brevi e nitide rappresentazioni schematiche, analoghe alle attività mentali elaborate all'inizio del primo ciclo di sonno (i cosiddetti microdreams: Nielsen, 2017). Per esempio, Kekulè descrisse così l'insight che lo portò nel 1861 alla scoperta dell'anello benzenico: "Ero seduto intento a scrivere, ma il lavoro non progrediva; i miei pensieri erano altrove. Girai la sedia verso il camino e mi appisolai. Gli atomi giocavano di fronte ai miei occhi (...) Il mio occhio mentale, reso più acuto da questa ripetuta visione, era ora in grado di distinguere strutture più grandi di multiforme conformazione; lunghe file talvolta sistemate più strettamente, tutte sinuose e ricurve come il moto di un serpente. Ma guarda! Uno dei serpenti aveva afferrato 
la sua stessa coda e la forma girava beffardamente davanti ai miei occhi. Come per un lampo improvviso mi risvegliai e passai il resto della notte a elaborare la mia ipotesi" (Morrison \& Boyd, 2002, p. 319).

È da rimarcare come dopo esperienze prolungate con attenzione focalizzata in videogiochi alcune parti dell'attività percettivo-motoria eseguita ricompaiano sotto forma di immagini più o meno brevi sia in addormentamento (Stickgold et al, 2000) che in sonno REM nella stessa notte (Wamsley et al., 2010). Inoltre, anche informazioni di tipo verbale trasmesse prima del sonno all'interno di un compito di memoria da completare dopo il risveglio vengono elaborate (cioè, scomposte nelle loro componenti semantiche e ricombinate con altre informazioni cui sono collegate da ben definite relazioni semantiche o episodiche, nonostante l'apparente bizzarria dei contenuti delle attività mentali: Cipolli et al., 1993) sia in stadio II NREM che in sonno REM (Cipolli et al., 1983, 1987). Infine, l'accesso alle informazioni verbali, che può essere iterato in stadi e cicli successivi di sonno (Cipolli et al., 1987), diventa selettivo (e quindi, specifico per il solo compito da risolvere). Infatti, se il compito viene variato dopo ogni risveglio provocato in diversi periodi di sonno REM della stessa notte, le informazioni incorporate tra $\mathrm{i}$ contenuti dell'attività mentale sono solo quelle relative al compito da risolvere proprio dopo quel risveglio (Cipolli et al., 2001, 2004). I dati di questi esperimenti dimostrano che durante il sonno la memoria viene utilizzata anche in senso prospettico, ovvero vengono riattivate anche le informazioni relative ad intenzioni di svolgere o risolvere compiti interrotti della veglia precedente (in tal caso dopo il risveglio successivo). In tal modo vengono ulteriormente consolidate in memoria non solo le informazioni rielaborate, ma anche le intenzioni in senso prospettico alle quali sono collegate (Barner et al., 2017). Appare quindi del tutto plausibile che questo complesso meccanismo sia attivo nella soluzione differita di un compito interrotto da completare dopo il sonno e, quindi, contribuisca, pur se non in modo esclusivo, a produrre l'effetto Zeigarnik (1927).

Numerose ricerche degli ultimi 15 anni realizzate con registrazione delle attività cerebrali sia con tecniche elettropoligrafiche che di neuroimaging (fMRIl) hanno dimostrato che le informazioni relative a compiti svolti intensivamente durante la veglia precedente sono riattivate non solo durante il sonno ma anche negli stati di resting (veglia rilassata: Feng et al., 2019) e mind wandering (attenzione non focalizzata: Chen et al., 2019). Le indicazioni così ottenute da una parte attestano la continuità (pur con variazioni di efficienza) tra diversi stati di vigilanza nel funzionamento di specifici processi cognitivi coinvolti nel pensiero creativo, dall'altra ne suggeriscono la complementarità, in quanto nella vita quotidiana questi processi vengono presumibilmente attivati tutti. Questa inferenza di 
ordine generale appare del tutto compatibile sia con le (auto)osservazioni più o meno aneddotiche di "creativi" (artisti e scienziati) che con osservazioni sui processi cognitivi attivati in condizioni di coscienza diverse dalla veglia attiva (con attenzione focalizzata), variamente definite come crepuscolari, ipnagogiche o simil-oniriche.

A quest'ultimo proposito merita di essere ricordato che Barolo aveva avviato nell'Istituto di Psicologia della Facoltà Medica di Milano un'indagine degli effetti indotti sulla soluzione di problemi dalla visualizzazione mentale nei flussi immaginativi compiuti a partire da elementi del campo problemico. I dati raccolti in alcuni esperimenti indicano che la libera visualizzazione di tipo "statico" agisce in senso facilitatorio o inibitorio a seconda delle caratteristiche del problema, mentre quella di tipo "dinamico" ha un'elevata potenzialità euristica qualunque sia la natura del problema. Tale effetto si realizza anche con flussi immaginativi prodotti in condizione ipnagogico-simile (Barolo, Antonietti, Cecchini e Stramba-Badiale, 1991). L'azione della visualizzazione mentale sembra consistere, quindi, non tanto nell'anticipazione di operazioni trasformative utili alla soluzione del problema, quanto piuttosto in una generale flessibilità del campo cognitivo da essa indotta, che porta a compiere operazioni analoghe a quelle che si riscontrano esaminando i contenuti delle attività mentali del sonno (Antonietti e Barolo, 1987).

\section{Conclusioni}

La distanza tra le suggestioni degli esperimenti sulla rêverie ipnagogica di Bertini (1980) e Foulkes e Fleisher (1975) e le evidenze attualmente disponibili sulla creatività in condizioni di mind wandering e resting (Gable et al., 2019), oltre che di sonno REM e NREM, è indubbiamente molto ampia. Tuttavia, se si esaminano retrospettivamente i lavori sperimentali sia eseguiti direttamente nel suo Istituto, sia stimolati da Cesa-Bianchi di persona negli ambiti congressuali ed editoriali, è possibile individuare un non trascurabile contributo della sua scuola in senso lato. Non va dimenticato, infatti, che la promozione editoriale, come è avvenuto tramite raccolte di studi in numeri monotematici di Ricerche di Psicologia, può essere una modalità decisiva per lo sviluppo delle conoscenze scientifiche. Questa modalità è tipica delle scuole, intese nell'accezione migliore della tradizione accademica, che possono promuovere aree di ricerca non soltanto al proprio interno o in collaborazione con studiosi di altre scuole, ma anche attraverso l'offerta di appropriate collocazioni editoriali per farne intuire le potenzialità. È perciò con piacere che si è colta la possibilità di 
pubblicare proprio su Ricerche di Psicologia questo ricordo del contributo di Marcello Cesa-Bianchi allo sviluppo di alcuni aspetti della psicologia del pensiero.

\section{Riferimenti bibliografici}

Albanese, O., Farina, E., \& Fiorilli, C. (2021). Cognizione, linguaggio, emozioni: ricerche evolutive e interventi educativi. Il contributo di Marcello Cesa-Bianchi e della sua scuola. Ricerche di Psicologia, 44(1), 167-176.

Amoretti, G. (2021). L'intelligenza e la sua misura. Il contributo di uno studioso eclettico: Marcello Cesa-Bianchi. Ricerche di Psicologia, 44(1), 63-76.

Anderson, J. R. (1978). Arguments concerning representations for mental imagery. Psychological Review, 85, 249-277.

Antonietti, A. (1991). Why does mental visualization facilitate problem-solving? In R. H. Logie \& M. Denis (Eds.), Mental images in human cognition ( 211227). Amsterdam: Elsevier.

Antonietti, A. (1999). Can student predict when imagery will allow them to discover the problem solution? European Journal of Cognitive Psychology, 11, 407-428.

Antonietti, A., \& Barolo, E. (1987). Pensiero onirico, immagini mentali e scoperte scientifiche. Ikon, 14, 121-140.

Antonietti, A., Barolo, E. \& Masini, R. (1985). Effetti prostrutturali, composizione di immagini mentali e problem-solving. Ricerche di Psicologia, 9 (1), 97125.

Antonietti, A., Barolo, E., \& Masini, R. (1986). Immagini mentali e stili cognitivi nella soluzione di problemi. Ikon, 13, 91-118.

Antonietti, A., Barolo, E., \& Masini, R. (1988). L'immagine mentale nella scoperta cognitiva. Contributi sperimentali. Milano: Edizioni Unicopli.

Antonietti, A., Bologna, D., \& Lupi G. (1997). The creative synthesis of visual images is not associated with individual differences. Perceptual and Motor Skills, 85, 881-882.

Antonietti, A., Cerana, P., \& Scafidi, L. (1994). Mental visualization before and after problem presentation: A comparison. Perceptual and Motor Skills, 78, 179-189.

Antonietti, A., \& Di Nuovo, S. (2014). Le applicazioni psicologiche della ricerca sulle immagini mentali. Introduzione al Forum. Ricerche di Psicologia, 85-87.

Barner, C., Seibold, M., Born, J., \& Diekelmann, S. (2017). Consolidation of prospective memory: Effects of sleep on completed and reinstated intentions. Frontiers in Psychology, 7, 2025.

Barolo, E. (1981). Organizzazioni alternative nello sviluppo cognitivo. La sfida della costruzione di immagini al pensiero logico-proposizionale. Milano: Edizioni Unicopli.

Barolo, E., Antonietti, A., Cecchini, I., \& Stramba-Badiale, P. (1991). Problemsolving ed elaborazioni iconiche in stato ipnagogico-simile. Ikon, 23, 199212 . 
Barolo, E., Masini, R., \& Antonietti, A. (1990). Mental rotation of solid objects and problem-solving in sighted and blind subjects. Journal of Mental Imagery, 14(3-4), 65-74.

Barolo, E., \& Stramba-Badiale, P. (Eds.) (1989). Pensiero visivo e psicoterapia. Saggi teorici e clinici sull 'analisi fantasmatica. Milano: Edizioni Unicopli.

Barrett, D. (1993). The "committee of sleep": A study of dream incubation for problem solving. Dreaming, 3, 115-122.

Barrett, D. (2001). The committee of sleep: How artists, scientists, and athletes use their dreams for creative problem solving and how you can too. New York: Crown Books/Random House.

Bertini, M. (1980). Una nuova tecnica per la rilevazione di contenuti mentali nel sonno. Ricerche di Psicologia, 4, 13-43.

Bertini, M., Lewis, H. B., \& Witkin, H. A. (1964). Some preliminary observations with and experimental procedure for the study of hypnagogic and related phenomena. Archivio di Psicologia, Neurologia e Psichiatria, 25, 493-534.

Bosinelli, M. (1980). Introduzione a "Processi mentali durante il sonno". Ricerche di Psicologia, 4, 7-12.

Cai, D. J., Mednick, S. A., Harrison, E. M., Kanady, J. C., \& Mednick, S.C. (2009). REM, not incubation, improves creativity by priming associative networks. Proceedings of the National Academy of Sciences-USA, 106, 1013010144.

Cai Y., Zhang, D., Liang, B., Wang, Z., Li, J., Gao, Z., Gao, M., Chang, S., Jiao, B., Huang, R., \& Liu, M. L. (2018). Relation of visual creative imagery manipulation to resting-state brain oscillations. Brain Imaging and Behavior 12, 258-273.

Chen, Q., Beaty, R. E., Cui, Z., Sun, J., He, H., Zhuang, K., Ren, Z., Liu, G., \& Qiu, J. (2019) Brain hemispheric involvement in visuospatial and verbal divergent thinking. Neuroimage, 202, 116065.

Cipolli, C. (1980). Processi di memoria e codificazione verbale nelle attività mentali del sonno: un approccio psicolinguistico. Ricerche di Psicologia, 4, 79-94.

Cipolli, C., Baroncini, P., Fagioli, I., Fumai, A., \& Salzarulo, P. (1987). The thematic continuity of mental sleep experience in the same night. Sleep, 10, 473479.

Cipolli, C., Bolzani, R., Cornoldi, C., De Beni, R., \& Fagioli, I. (1993). Bizarreness effect in dream recall. Sleep, 16, 163-170.

Cipolli, C., Bolzani, R., Tuozzi, G., \& Fagioli, I. (2001). Active processing of declarative knowledge during REM-sleep dreaming. Journal of Sleep Research, 10, 277-284.

Cipolli, C., Cicogna, P. C., Mattarozzi, K., Mazzetti, M., Natale, V., \& Occhionero, M. (2003). Continuity of the processing of declarative knowledge during human sleep: evidence from interrelated contents of mental sleep experiences. Neuroscience Letters, 342, 147-150.

Cipolli, C., Fagioli, I., Maccolini, S., \& Salzarulo, P. (1983). Associative relationships between pre-sleep sentence stimuli and reports of mental sleep experience. Perceptual and Motor Skills, 56, 223-234. 
Cipolli, C., Fagioli, I., Mazzetti, M., \& Tuozzi, G. (2004). Incorporation of presleep stimuli into dream contents: evidence for a consolidation effect on declarative knowledge during REM sleep? Journal of Sleep Research., 13, 317-326.

Cipolli, C., Fagioli, I., Mazzetti, M., \& Tuozzi G. (2006). Consolidation effect of repeated processing of declarative knowledge in mental experiences during human sleep. Brain Research Bulletin, 69, 501-511.

Cornoldi, C. (1976). Memoria e immaginazione. Bologna, Casa Editrice Pàtron.

Cristini, C., Cesa-Bianchi, G., \& De Beni, R. (2021). Psicologia dell'invecchiamento e dell'età longeva: il contributo di Marcello Cesa-Bianchi. Ricerche di Psicologia, 44(1), 177-190.

Di Nuovo, S. (Ed.) (1999). Mente e immaginazione: la progettualità creativa in educazione e terapia. Milano: FrancoAngeli Editore.

Feng, Q., He, L., Yang, W., Zhang, Y., Wu, X., \& Qiu, J. (2019). Verbal creativity is correlated with the dynamic reconfiguration of brain networks in the resting state. Frontiers in Psychology, 10, 894.

Foulkes, D. (1962). Dream reports from different stages of sleep. Journal of Abnormal and Social Psychology, 65, 14-25.

Foulkes, D., \& Fleisher, S. (1975). Mental activity in relaxed wakefulness. Journal of Abnormal Psychology, 84, 66-75.

Foulkes, D., \& Rechtschaffen, A. (1964) Presleep determinants of dream content: Effects of two films. Perceptual and Motor Skills, 19, 983-1005.

Gable, S. L., Hopper, E. A., \& Schooler, J. W. (2019) When the Muses strike: Creative ideas of physicists and writers routinely occur during mind wandering. Psychological Science, 30, 396-404.

Gladwell, M. (2007). Blink: The power of thinking without thinking. Back Bay Book, 2007, second edition (trad. it. In un batter di ciglia. Mondadori, Milano, 2019).

Incorpora, C., Oliveri, S., Genevini, M., Santagostino, L., Tettamanti, L., Antonietti, A., \& Risoli, A. (2010). Visual and verbal styles in patients with acquired brain damage and cognitive deficits: A preliminary study. International Journal of Neuroscience, 120, 557-564.

Inghilleri, P. (Ed.) (2009). Psicologia culturale. Milano: Raffaello Cortina Editore.

Kosslyn, S. M. (1980). Image and mind. Cambridge: Harvard University Press.

Kramer, M., \& Roth, T. (1979). The stability and variability of dreaming. Sleep, 1, 319-325.

Leong, R. L. F., Cheng, G. H., Chee, M. W. L., \& Lo, J. C. (2019). The effects of sleep on prospective memory: A systematic review and meta-analysis. Sleep Medicine Reviews, 47, 18-27.

Leszczynski, M., Chaieb, L., Reber, T. P.Derner, M., Axmacher, N., \& Fell, J. (2017). Mind wandering simultaneously prolongs reactions and promotes creative incubation. Scientific Reports, 7, 10197.

Lewis, P. A., Knoblich, G., \& Poe, G. (2018). How memory replay in sleep boosts creative problem-solving. Trends in Cognitive Sciences, 22, 491-503. 
Li, W., Yang, J., Zhang, Q., Li, G., \& Qiu, J. (2016). The association between resting functional connectivity and visual creativity. Scientific Reports, 6, 25395.

Masini, R. (1979). Immagini e parole. Contributi sperimentali. Milano: Edizioni Unicopli.

Mehler, J., \& Bever, T. G. (1972). Editorial. Cognition, 1, 9-11.

Morrison, R. T., \& Boyd, R. N. (2002). Organic chemistry (6 $6^{\text {th }}$ Edition). New Delhi: Prentice-Hall of India.

Nielsen, T. A. (2004). Chronobiological features of dream production. Sleep Medicine Reviews, 8, 403-424.

Nielsen, T. A. (2017). Microdream neurophenomenology. Neuroscience of Consciousness, 2, 1-17.

Oldrati, V., Colombo, B., \& Antonietti, A. (2018). Combination of a short cognitive training and tDCS to enhance visuospatial skills: A comparison between online and offline neuromodulation. Brain Research, 1678, 31-39.

Rechtschaffen, A., Vogel, G., \& Shaikun, G. (1963). Interrelatedness of mental activity during sleep. Archives of General Psychiatry, 9, 536-547.

Salzarulo, P., \& Cipolli, C. (1974). Spontaneously recalled verbal material and its linguistic organization in relation to different stages of sleep. Biological Psychology, 2, 47-57.

Salzarulo, P., \& Cipolli, C. (1979). Linguistic organization and cognitive implications of REM and NREM sleep-related reports. Perceptual and Motor Skills, 49, 767-777.

Schoch, S. F., Cordi, M. J., Schredl, M., \& Rasch, B. (2019). The effect of dream report collection and dream incorporation on memory consolidation during sleep. Journal of Sleep Reserarch, 28, e12754.

Shepard, R. N. (1975). Form, formation, and transformation of internal representations. In A. A. Sheikh \& J. T. Shaffer (Eds.), The potential in fantasy and imagination (279-341). Hillsdale, NJ: Lawrence Erlbaum Associates.

Snyder, F. (1970). The phenomenology of dreaming. In L. Madow \& L. Snow (Eds.). The psychodynamic implications of the physiological studies on dreams (124-151). Springfield, Illinois: Thomas.

Stickgold, R., Malia, A., Maguire, D., Roddenberry, D., \& O’Connor, M. (2000). Replaying the game: Hypnagogic images in normals and amnesics. Science, 290 (5490), 350-353.

Stickgold, R., Scott, L., Rittenhouse, C., \& Hobson, J. A. (1999). Sleep-induced changes in associative memory. Journal of Cognitive Neuroscience, 11, 182193.

Tassi, P., \& Muzet, A. (2000). Sleep inertia. Sleep Medicine Reviews, 4, 341-353.

Thompson, A. L., \& Klatzky, R. L. (1978). Studies of visual synthesis: Integration of fragments into forms. Journal of Experimental Psychology: Human Perception \& Performance, 4, 244-263.

Wagner, U., Gais, S., Haider, H., Verleger, R., \& Born, J. (2004) Sleep inspires insight. Nature, 427 (6972), 352-355. 
Walker, M. P., Liston, C., Hobson, J. A., \& Stickgold, R. (2002). Cognitive flexibility across the sleep-wake cycle: REM-sleep enhancement of anagram problem solving. Brain Research and Cognive Brain Research 14, 317-324.

Wamsley, E. J., Perry, K., Djonlagic, I., Reaven, L. B., \& Stickgold, R. (2010). Cognitive replay of visuomotor learning at sleep onset: Temporal dynamics and relationship to task performance. Sleep, 33, 59-68.

Zeigarnik, B. (1927). Ueber das Behalten von erledigten und everledigten Handlungen. Psychologische Forschungen, 9, 1-85. 